\title{
MALDI Mass Spectrometry Imaging of Neuronal Cell Cultures
}

\author{
Tyler A. Zimmerman, ${ }^{1,2}$ Stanislav S. Rubakhin, ${ }^{1,2}$ Jonathan V. Sweedler ${ }^{1,2}$ \\ ${ }^{1}$ Department of Chemistry, University of Illinois, 600 South Mathews Ave.; 63-5, Urbana, IL 61801, USA \\ ${ }^{2}$ Beckman Institute, University of Illinois, Urbana, IL 61801, USA
}

\begin{abstract}
Mass spectrometry imaging (MSI) provides the ability to detect and identify a broad range of analytes and their spatial distributions from a variety of sample types, including tissue sections. Here we describe an approach for probing neuropeptides from sparse cell cultures using matrixassisted laser desorption/ionization time-of-flight (MALDI-TOF) MSI-at single cell spatial resolution-in both MS and tandem MS modes. Cultures of Aplysia californica neurons are grown on an array of glass beads embedded in a stretchable layer of Parafilm $M$. As the membrane is stretched, the beads/neurons are separated physically and the separated beads/ neurons analyzed via MALDI TOF MS. Compared with direct MS imaging of samples, the stretching procedure enhances analyte extraction and incorporation into the MALDI matrix, with negligible analyte spread between separated beads. MALDI tandem MSI using the stretched imaging approach yields localization maps of both parent and fragment ions from Aplysia pedal peptide, thereby confirming peptide identification. This methodology represents a flexible platform for MSI investigation of a variety of cell cultures, including functioning neuronal networks.
\end{abstract}

Key words: MSI, MALDI, Aplysia, Cell cultures

\section{Introduction}

$\mathrm{E}$ xperiments using cell cultures are widespread in fundamental [1, 2], applied [3, 4], and clinical/diagnostic [5-7] research. Detection of known and unknown endogenous analytes and determination of their levels at cellular and subcellular resolutions aid many experiments involving cell cultures. As with biological tissues, analyte detection in cultured cells utilizes a variety of targeted approaches that include immunocytochemistry [8], molecular information-rich spectroscopic methodologies such as infrared spectroscopy [9, $10]$, and mass spectrometry (MS) $[11,12]$, each having

Electronic supplementary material The online version of this article (doi:10.1007/s13361-011-0111-2) contains supplementary material, which is available to authorized users.

Correspondence to: Jonathan V. Sweedler; e-mail: jsweedle@illinois.edu inherent drawbacks and benefits. Immunochemistry, for example, requires pre-selection of the analyte(s) of interest; in contrast, MS allows identification of a range of analytes without pre-selection, but does not provide spatial and localization details. However, spatial information can be obtained from cell cultures by combining MS with imaging technologies, specifically, mass spectrometry imaging (MSI).

MSI has emerged as a powerful tool for investigation of biological specimens; it simultaneously provides chemical and spatial distribution information on many analytes [1318]. Secondary ion mass spectrometry (SIMS) was the first MS-based imaging approach [19], and is capable of investigating chemically complex surfaces such as tissue slices or cell cultures with submicron spatial resolution [20, 21]. Unfortunately, the limited mass range of SIMS restricts its ability to detect many peptides and proteins. Matrixassisted laser desorption/ionization (MALDI) MS extends the mass range and is well suited for localizing larger

Received: 27 September 2010

Revised: 21 February 2011

Accepted: 23 February 2011

Published online: 26 March 2011 
analytes to specific cultured cells [22-25], as are several other laser desorption approaches [26, 27]. MALDI MSI has also been used for profiling the spatial distributions of analytes in cultured cells [23, 28, 29]. In MALDI MSI, data acquisition is often performed using an ordered array of positions to allow the creation of two-dimensional ion distribution maps [30], although other data acquisition approaches are available [31-33]. When seeking single cell resolution from MALDI MSI investigations of peptides, challenges can arise due to several factors. These include the inherent biochemical complexity of the cell cultures, the close and random proximity of adjacent cells, significant differences in peptide content among neighboring cells, the possibility of mechanical damage to cells during removal of the extracellular media, and the small amount of peptide present in many cell types. When a peptide is near the detection threshold in MSI, the amount per pixel is often in the low attomole range, an estimate based on prior Aplysia microanalyses [34, 35]. We address many of these issues by using a sample preparation technique known as the stretched imaging method [36-38], which has been applied to characterize peptide distributions in tissue slices from both invertebrate and mammalian models. Briefly, a thin tissue slice is placed onto a monolayer of beads embedded in a stretchable layer of Parafilm M. This substrate is manually stretched and as the beads separate from each other, the tissue is fragmented into thousands of spatially and chemically isolated bead islands that contain one or a few cells [37]. After stretching, the hydrophobic nature of the intervening membrane between bead/cell positions prevents analyte redistribution when the MALDI matrix is applied, allowing extended extraction periods [30]. This separation of cells permits small, spatially isolated samples to be characterized, at the same time offering increased analyte extraction from the tissue, thereby providing enhanced analyte signals. The stretched imaging protocol thus minimizes the potential difficulties outlined above for MALDI MSI experiments.

For this study, we created MSI images from cultured Aplysia californica neurons with well-characterized peptide contents $[34,36,39]$ that allow protocol validation; future applications will examine a broader range of samples. Although MSI can generate chemical and spatial information from analytes in cell cultures, it is sometimes difficult to identify compounds detected from a cell with single stage MS [35]. It is therefore helpful to employ tandem MS (MS/ MS) for analyte identification, including de novo peptide sequencing. The success of MS/MS sample fragmentation depends on the amount of analyte available for analysis. The stretched imaging method aids the investigation by allowing independent optimization of the imaging parameters as well as enhancing the analyte extraction and MALDI matrix incorporation process, thus allowing the MS/MS experiments to be tailored for small-volume samples. Here, we used the method to generate both MS and MS/MS reconstructed ion images of high quality from Aplysia neuronal cultures. The ability to provide chemical, spatial, and amino acid sequence information via automated image acquisition makes the newly adapted stretched imaging method well suited for the study of cell cultures from a variety of model organisms.

\section{Experimental}

\section{Chemicals}

Reagents used in the study were from Sigma-Aldrich, St. Louis, MO, USA, except where specifically noted.

\section{Substrate Creation and Cell Isolation}

Substrates comprised of a hexagonally-packed layer of glass beads embedded in a Parafilm M layer (Pechiney, Neenah, WI, USA) were created as previously described [37], with the exception that the $\sim 40 \mu \mathrm{m}$ glass beads (Mo-Sci Corp., Rolla, MO, USA) used here were made from borosilicate instead of barium/titanium. The borosilicate beads on the substrate were coated with poly-L- or poly-D-lysine. Individual neurons were isolated from the Aplysia central nervous system after its dissection and enzymatic treatment with protease IX for $30-60 \mathrm{~min}$ at $34{ }^{\circ} \mathrm{C}$. The neurons were distributed onto the bead array. The cell culture was allowed to develop overnight at $14{ }^{\circ} \mathrm{C}$ in artificial sea water (ASW) containing (in $\mathrm{mM}$ ): $460 \mathrm{NaCl}, 10 \mathrm{KCl}, 10 \mathrm{CaCl}_{2}, 22$ $\mathrm{MgCl}_{2}, 6 \mathrm{MgSO}_{4}$, and $10 N$-2-hydroxyethylpiperazine- $N^{\prime}-2$ ethanesulfonic acid (HEPES) (pH 7.8) [37] supplemented with antibiotics: 100 units $/ \mathrm{mL}$ penicillin $\mathrm{G}, 100 \mu \mathrm{g} / \mathrm{mL}$ streptomycin, and $100 \mu \mathrm{g} / \mathrm{mL}$ gentamicin and a saturated concentration of amphotericin B.

\section{Sample Preparation for Environmental Scanning Electron Microscopy}

Visualization of neurites and other small features such as regions of contact between the cells and beads using conventional transmission or reflection light microscopy is difficult when cell cultures are developed on a Parafilm M-bound glass bead array. Thus, environmental scanning electron microscopy (ESEM) was employed to image cultured cells on the bead array because it provides the level of spatial resolution needed to observe neurites. For ESEM, cell cultures were fixed using a $4 \mathrm{~h}$ treatment of a mixture of $2.5 \%$ glutaraldehyde and $2 \%$ paraformaldehyde in $0.1 \mathrm{M}$ sodium cocadilate buffer. The samples were then washed for $15 \mathrm{~min}$ in $0.1 \mathrm{M}$ sodium cocadilate buffer and dehydrated in a series of $37 \%, 67 \%, 95 \%$, and $100 \%$ ethanol solutions. A Samdri-PVT-3D critical point dryer (Tousimis Research Corp.; Rockville, MD, USA) was employed to remove the ethanol from the samples. The samples were removed from the critical point dryer and placed onto twosided tape (3 M Corporation, St. Paul, MN, USA), which was then mounted onto a sample stage. A Desk II Turbo 
Sputter Coater (Denton Vacuum, Moorestown, NJ, USA) was used to coat the samples with $\mathrm{Au} / \mathrm{Pd}$ for $65 \mathrm{~s}$, achieving $\sim 15 \mathrm{~nm}$ thickness of the metal layer. The samples were then placed in a desiccator overnight. ESEM images were taken with an XL30-ESEM-FEG (FEI, Hillsboro, OR, USA) scanning electron microscope that includes a field-emission electron gun.

\section{Sample Preparation for MSI}

Cell cultures on the bead substrates were stabilized for 10 20 min by replacement of the ASW with a 33\% glycerol: $67 \%$ ASW (vol:vol) solution, followed by removal of the extracellular media. A small amount of the glycerol/ASW solution remained on the samples, which is acceptable for MALDI MS detection of peptides. The directional orientation of the samples before stretching was marked with a magic marker. Digital optical images were obtained via a stereo microscope (Leica Microsystems, Bannockburn, IL, USA), followed by manual stretching of the samples and placing them on conductive indium tin-oxide (ITO)-coated glass slides as previously reported [36]. The glass slides were inserted into a Bruker MTP Slide Adapter (Bruker Daltonics, Billerica, MA, USA). Alternatively, samples used for acquiring both MS and MS/MS images were stretched and placed onto metal TLC MALDI plates (Bruker Daltonics).

Immediately following, a MALDI matrix containing $30 \mathrm{mg} / \mathrm{mL}$ of 2,5-dihydroxybenzoic acid and 50:50 methanol/water was applied to the surface of the stretched samples using an artist's spray brush (Thayer and Chandler, Kenosha, W, USA) at a distance of $\sim 30 \mathrm{~cm}$, as in a manner previously reported [40]. For samples placed on the ITO glass slides, after matrix application a razor blade was used to remove the Parafilm $M$ from the surface near the edges of the slide to improve electrical contact with the metal MTP Slide Adapter (Bruker Daltonics). Optical images were taken of all samples after stretching. Where multiple images were needed to capture the entire sample area, these subsidiary images were stitched together using Photoshop CS2 software (Adobe Systems, San Jose, CA, USA).

\section{MSI of Stretched Samples}

For the samples placed on the glass ITO slides, the bead positions after sample stretching were automatically located by light thresholding, and an in-house written Java-based (http:// java.sun.com) geometry file creation code was used to create sample-tailored geometry files [36]. For metal plates, transmission-based light thresholding was not possible and, so, the cells were located manually, the cell pixel coordinates recorded, and the bead location geometry file creation code modified to correspond to cell locations and the dimensions of the Bruker metal TLC plate adaptor. The original and modified source codes are freely available at http://neuroproteomics.scs. illinois.edu/imaging.html.
The mass spectrometric acquisition was automated using the geometry files loaded into the AutoXecute feature of FlexControl 3.0 software (Bruker Daltonics) on an UltrafleXtreme MALDI time-of-flight (TOF)-TOF MS instrument (Bruker Daltonics), with the laser spot size set to $\sim 100 \mu \mathrm{m}$ in diameter. For samples on ITO glass slides, at each position 100 spectra were accumulated at $1000 \mathrm{~Hz}$ using a mass range of 600-4000 Da. For samples on the solid metal targets, 100 spectra were accumulated at each point and 400 spectra accumulated in Bruker LIFT mode (MS/MS) at $1000 \mathrm{~Hz}$ at the points for which peaks were automatically selected by the software for MS/MS acquisition. Parent ions were automatically selected and fragmented in LIFT mode. The parameters of the process were specified in a FlexAnalysis (Bruker Daltonics) method loaded into AutoXecute in the Bruker FlexControl 3.0 software. The thresholds for accepting detected signals were a peak intensity of greater than 400 counts and a signal-to-noise ratio of better than 10 . Mass calibration was done with Peptide Calibration Standard II (Bruker Daltonics). For samples on the glass slides, the entire area containing $\sim 1200$ beads was imaged in $\sim 40 \mathrm{~min}$. For samples on the metal targets, only positions containing cells were interrogated so that acquisition time was greatly reduced; the time to acquire MS and MS/MS spectra from a total of 28 cell positions was 6 min.

The spectra in the Bruker fid files were baseline subtracted, smoothed, and converted into spectra-containing text files using a batch conversion script written in the FlexAnalysis 3.3 software (Bruker Daltonics). These files were then able to be read by the previously described Javabased image reconstruction software [36]. For all samples, this code created selected MS ion images based on the $\mathrm{m} / \mathrm{z}$ ratio of the analyte of interest. For the samples on the metal target, the code was modified to produce MS/MS ion images. The original image reconstruction portion of the code was unaltered, but the portion that creates the ion images was adapted to produce reconstructed MS/MS ion images. This code is freely available at http://neuroproteomics. scs.illinois.edu/imaging.html.

The MS/MS results for the peptide parent ion at $m / z 1540$ were analyzed by BioTools 3.0 (Bruker Daltonics), where the assignment of the amino acid sequence was generated automatically and searched against a peptide database using Mascot software (Matrix Science Inc., Boston, MA, USA) to reveal the identity of the peptide. Collections of prohormone genes from a variety of model organisms including Aplysia are available at http://neuroproteomics.scs.illinois.edu/neuropred/ sequences/Sequencedata.html

\section{Results and Discussion}

A variety of MALDI MSI experiments can be devised to probe neuronal cultures. By combining MALDI-MSI with the stretched imaging method, we can investigate sparse neuronal cell cultures at cellular (and we believe eventually at subcellular) levels to provide details on peptide distribu- 
tions and changes in their expression during the formation of networks. These investigations may also shed light on network formation and repair, as well as changes in the distributions of peptides [23, 41, 42]. However, to accomplish these neuronal stretched imaging experiments, several enhancements to the existing stretched imaging protocols were necessary. As detailed below, these included a switch to poly-lysine-coated borosilicate beads so that the cells would attach to the substrate and grow on it, the use of a metal MALDI target to reduce charging and enable MS/MS imaging, high spatial resolution ESEM verification of cellbead attachment for cultured neurons, and updated software to allow automated runs of the MS and MS/MS imaging. The optimized stretched imaging method, applied here to a sample of cultured Aplysia neurons, facilitates the creation of reconstructed ion images that show the distributions of neuropeptides and peptide fragments.

Initially, when creating the bead substrate and shortly after plating, the Aplysia neurons detached from the barium/ titanium glass beads previously used with the stretched sample method for $\sim 20-\mu \mathrm{m}$-thick tissue sections $[36,37]$. Alternatively, we found that $\sim 40 \mu \mathrm{m}$ borosilicate glass beads coated with poly-L- or D-lysine placed onto the Parafilm M layer provided an improved substrate for neuronal growth; the cultured neurons remained attached to this substrate, an expected result given the reported use of borosilicate [43$45]$ and lysine coating [46, 47] for neuronal cell culture. Not only is the composition of this substrate ideal for the cells to attach and grow, so also is the relatively uniform threedimensional surface of the bead monolayer with patterned topography. The extending processes of cultured neurons respond favorably to topographic cues on this scale [48-50] and, so, culturing neurons on beads served to enhance neuronal growth.

After the neuronal cultures were developed on the new bead substrate, we verified that the cells were attaching to the beads; attachment is necessary for successful spatial separation of cultured cells as the underlying support membrane is stretched. Previously, we used optical microscopy to visualize the attachment of neurons and their processes to the beads. However, ESEM was employed here because it can reveal smaller features such as neurites and growth cones of cultured neurons. ESEM confirmed that the curved topography of the lysine-coated borosilicate bead monolayer provided a suitable substrate along which the cultured Aplysia neurons could extend their processes (Figure 1). As seen in Figure 1a, the neuron and its neurites, (i.e., projections) are well attached to the beads. There are extending zones of adhesion atop the beads, including a growth cone-like structure terminating the neurites. The neurites often covered multiple beads with many smaller extensions (filopodia), with some forming fine submicrometer bridges to neighboring beads. Intriguingly, smaller neurites that spanned the gaps between beads did so at positions where the beads were not touching, indicating these outgrowths likely "jumped" from bead to bead. Small, $\sim 2 \mu \mathrm{m}$ enlargements in the extending processes, often termed varicosities [51] (putatively containing secretory vesicles), have been detected along the smallest branches that stem off from the main processes. Manual sample stretching separates adjacent beads and cells, and may also separate the neurites and varicosities from the cell bodies during the stretch. While single cell measurements are possible - because no more than a single cell appears on most beads-subcellular MALDI MSI measurements may also be possible using this approach. However, development of additional verification steps will be required before identification of subcellular origins of signal can be achieved.

Aplysia neurons from both the pedal and cerebral ganglia were used to extend the stretched imaging method to MSI investigation of cell cultures. An optical image was taken of the cell cultures before stretching (Figure 2a). Several cell bodies are visible in the image, with some neurons located in close proximity to each other. Overlays of three reconstructed ion images (Figure 2b-d) onto the initial sample image show minimal analyte redistribution and that the ion signals are confined to the area of cell somas (i.e., cell bodies); the image of the $\mathrm{m} / \mathrm{z} 635$ ion distribution (presumably a membrane lipid, Figure 2b) contains a signal strong

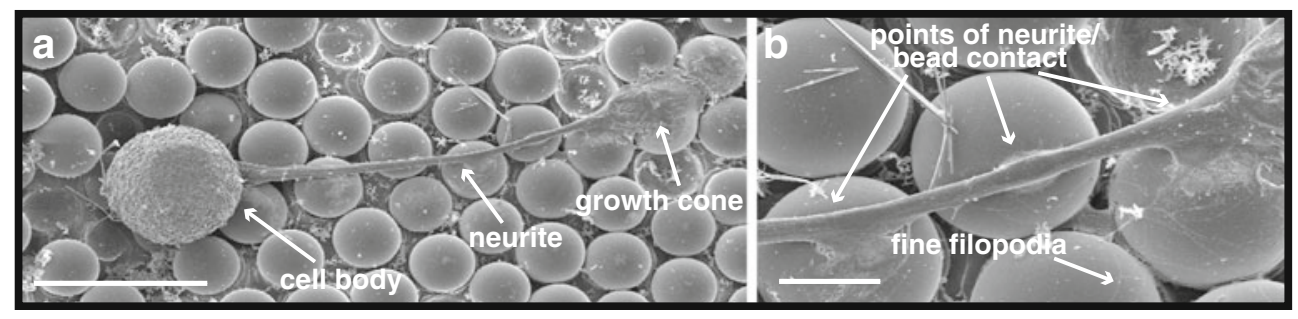

Figure 1. Environmental scanning electron microscopy (ESEM) imaging of cultured Aplysia neurons and neurites on the bead array reveals the attachment and growth of neurons on this topologically complex surface. (a) A cultured neuron is attached to beads that are embedded in a layer of Parafilm $\mathrm{M}$. The neuronal process extends away from the cell body over the length of several beads. The sample preparation procedure for ESEM caused some beads to be removed from the sample surface. The cell body, extending neurite, and growth cone are labeled. Scale bar is $100 \mu \mathrm{m}$. (b) Close-up view of the center region of the left image. The sample stretching may lead to mechanical separation of subcellular regions, which would be available for analysis using MSI. The points of neurite-bead contact and the fine filopodia are labeled. Scale bar is $20 \mu \mathrm{m}$ 

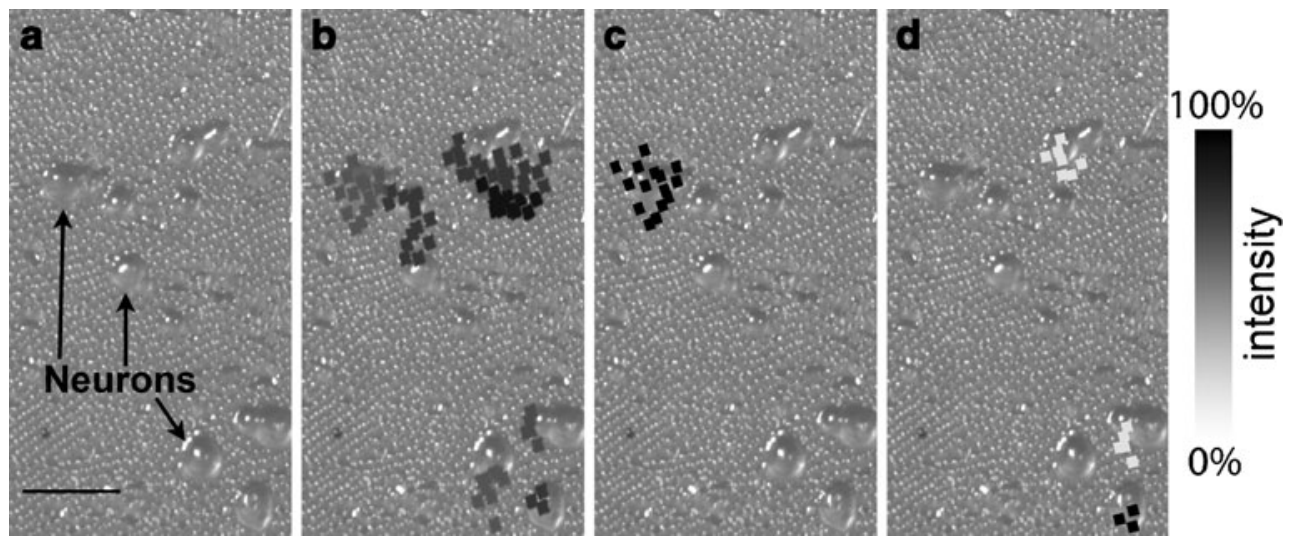

Figure 2. Overlaid optical and reconstructed ion images created by the stretched imaging method from a neuronal culture from different ganglia of Aplysia CNS. About 1200 total beads were probed. (a) An optical image of the sample showing the cultured neurons on a substrate of borosilicate glass beads embedded in a Parafilm M layer. In (b)-(d), the reconstructed ion images overlay the original optical image to show the selected analyte distributions, with (b) showing $\mathrm{m} / \mathrm{z} 635$, (c) $\mathrm{m} / \mathrm{z} 1238$, and (d) $\mathrm{m} / \mathrm{z}$ 1540, which have been assigned as a membrane lipid, FRFa peptide, and pedal peptide, respectively. The squares represent the bead positions that have the appropriate $\mathrm{m} / \mathrm{z}$ signal with their grey color matching the intensity scale bar on the right. In each image, the intensity has been scaled to the maximum level. The analyte distributions are confined to individual cell somas in many cases. Scale bar is $500 \mu \mathrm{m}$

enough to highlight locations of the majority of the cell somas within the field of view. Two of the most intense detected ion signals are assigned to well-known Aplysia peptides, FRFa [GenBank accession number GU263310] (Figure 2c) and pedal peptide [accession number AAV84473.1] (Figure 2d). Many of the signals from individual cells have similar intensities (Figure 2b), which is not surprising as cells may have similar peptide levels and many of the studied neurons are of similar size.

How are the peptide identifications made? Typically, the observed peptide signals from an MSI image are first tentatively identified based on their $\mathrm{m} / \mathrm{z}$ and compared with lists of known peptides, as well as from co-localization with other peptides known to be expressed in these cells, such as multiple peptides derived from the same prohormone. For example, an MSI spectrum from a cultured neuron sample is shown in Figure S1, Supplementary Information (on-line resource). Signals can then be identified using MS/MS, either off-line with liquid chromatography-MS/MS of homogenates or, in this case, during the same experiment via MALDI-MS/MS imaging.

The ability to identify peptides by MS/MS sequencing allows peak assignments to be confirmed [52-54]. Several adjustments to the stretched sample method enable MALDIMS/MS sequencing from stretched samples of cultured neurons; these include the use of a metal MALDI target and adapting the stretched imaging software to facilitate automated MS/MS imaging runs. The use of a metal MALDI target had not been necessary as ITO slides allowed peptide signals to be detected from stretched neuronal cultures in MS mode (Figure 3). However, ITO slides did not work for automated acquisition of MS/MS spectra. Attempts to reproducibly acquire MS/MS spectra from stretched samples on ITO slides led to unreliable accuracy of the parent ion $\mathrm{m} / \mathrm{z}$ measurements. Shifts of up to $30 \mathrm{Da}$ in the $m / z$ ratio were observed, even after increasing the sample conductivity with a thin metal coating [55]. These mass calibration issues prevented automated ion fragmentation. However, placing the stretched sample onto a solid metal Bruker TLC MALDI plate ameliorated these issues, possibly because of the increased electrical conductivity and improved heat dissipation afforded by the solid metal target.

The geometry file creation and image reconstruction software, as created for imaging of stretched thin tissue sections [36], has been adapted here for MS/MS profiling of stretched samples containing cultured neurons on metal plates. For the ITO glass slides, light thresholding was used with the optical images, allowing the automatic determination of the X-Y coordinates of the beads with this information used to create a geometry file containing the locations where the MS spectra were to be collected. However, when using optically opaque metal plates for MS/MS imaging, transmission-based optical imaging of the samples was not possible. As a future enhancement, it may be possible to use light thresholding for the creation of geometry files on metal targets via reflection-based optical images; here, we obtained MS/MS data from cell somas at manually determined positions. As currently implemented, the new target geometry file now works with the Bruker TLC targets and enables one to obtain MS/MS spectra from preselected cell somas. This software is easily adaptable to a range of other targets and applications. As before, acquisition of the MS/MS spectra from the specified locations precedes image reconstruction using the free transform process in Photoshop [36]. Lastly, the image reconstruction code now reads data from the MS/MS spectra generated by the Bruker mass spectrometer in LIFT mode. The software, with graphical user interfaces, is freely available online along with example datasets and step-by-step guidance for 


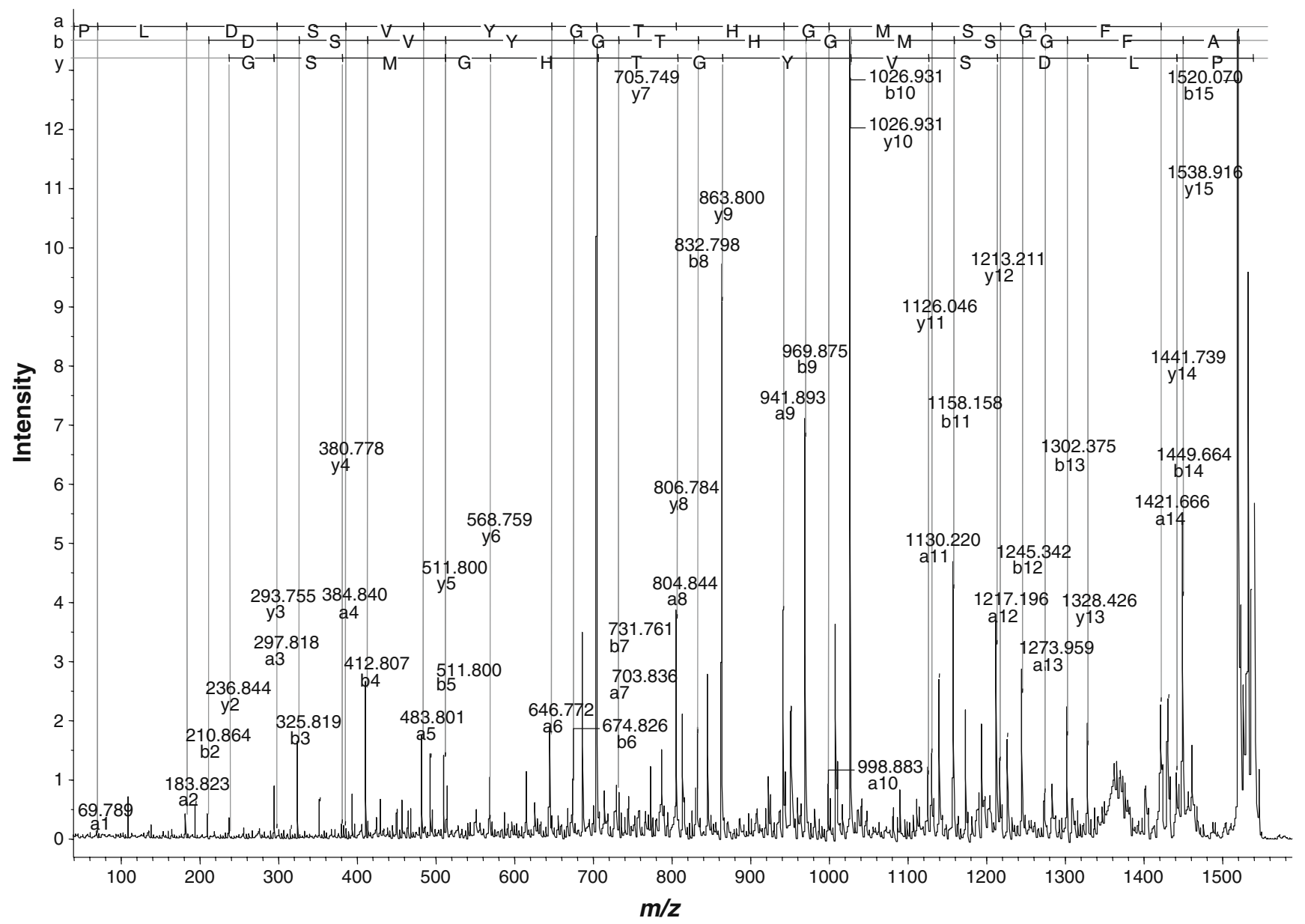

Figure 3. MALDI-TOF detects strong neuropeptide signals in MS and MS/MS modes from cell culture samples prepared using the stretched imaging method. The mass spectrum of the peptide profile of a selected cultured pedal neuron is shown; the automatically generated MS/MS peak assignment for $\mathrm{m} / \mathrm{z} 1540$ yields the sequence that corresponds to the known amino acid sequence for Aplysia pedal peptide, PLDSVYGTHGMSGFA

geometry file creation at http://neuroproteomics.scs.illinois. edu/imaging.html.

Figure 3 shows an MS/MS spectrum from a stretched sample of cultured Aplysia neurons placed onto the solid metal MALDI plate, as shown for the parent mass ion of $\mathrm{m} / \mathrm{z}$ 1540. This MS/MS spectrum was taken from an automated imaging run and is labeled with de novo peak assignments generated by the Bruker BioTools 3.0 software. Most peaks corresponded to fragments of Aplysia pedal peptide. Loading of the results into the Mascot search engine produced a hit with a highest Mascot score of 112 for Aplysia pedal peptide. The quality of the MS/MS spectra automatically acquired from single cells using the stretched imaging method allows for sequencing and identification of peptides with high confidence from a sample of cultured neurons. In addition, the distributions of the parent peptide and its fragment peptides are able to be simultaneously mapped.

Earlier in this study, the stretched imaging method was used to acquire MS images from all positions containing beads (Figure 2). We also imaged selected regions from a different stretched sample that contained neurons, to obtain both MS and MS/MS ion profiles. These reconstructed MALDI-MS/MS ion distribution maps are shown in Figure 4. Because the locations of the neuron somas were easily visible in the optical images in Figure 4 taken both before and after stretching, their locations were manually recorded. Subsequently, a geometry file containing only these cell positions was used to guide the acquisition of MS and MS/ MS mode ion images solely at the cell locations.

A similar approach can be applied for characterization and imaging of specific peptide(s) of interest after MSI by first manually creating a mass list of detected peptide parent ions with corresponding spatial coordinates and then optimizing the MS/MS parameters and precursor ion selection window for the analyte(s) of interest for the subsequent MS/MS imaging scan. An advantage to only imaging the beads that contain neurons (and not empty beads or membrane) is a significant reduction in image acquisition time and resulting data. MALDI-MSI with the specific TOF-TOF instrumental platform used here does not allow rapid transitions from MS to MS/MS mode. Therefore, independent scans in MS and MS/MS modes were 

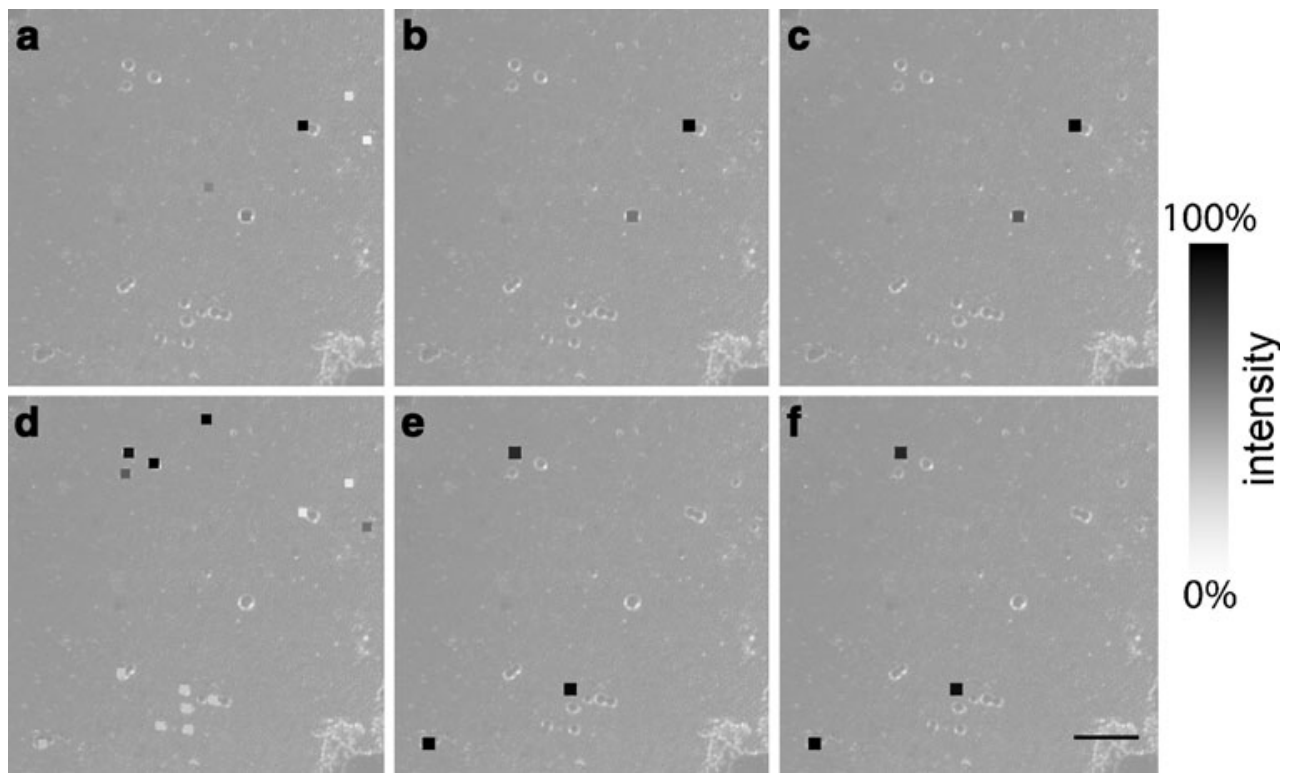

Figure 4. Reconstructed MS and MS/MS ion images of $\mathrm{m} / \mathrm{z} 1540$ and 1200 parent ions. The background is an optical image of the sample taken before stretching where the cultured neurons are visible and labeled, with the gray/black boxes representing the MS ion image data (i.e.; cells). (a) Reconstructed MS ion image of parent ion $\mathrm{m} / \mathrm{z} 1540$ that corresponds to pedal peptide. Reconstructed MS/MS ion images of pedal peptide fragments ions are shown in (b) $\mathrm{m} / \mathrm{z} 419$ and (c) $\mathrm{m} / \mathrm{z} 1245$. (d) Reconstructed MS ion image of parent ion $\mathrm{m} / \mathrm{z} 1200$ corresponding to a putative but unidentified peptide. Reconstructed MS/MS fragment ion images are shown in (e) $\mathrm{m} / \mathrm{z} 219$ and (f) $\mathrm{m} / \mathrm{z} 922$ [fragments in (e) and (f) are currently unidentified]. Only some neurons were selected for acquisition of MS/MS spectra because not all neurons contain the analyte of interest. Scale bar is $500 \mu \mathrm{m}$

performed in tandem, thereby reducing the time for complete MS imaging and analyte identification. The stretched imaging method does not deplete the sample significantly during acquisition, even from individual cells under culture conditions, thus allowing repeat scans. Depending on the needs of the experiment, sample locations containing peptides of interest can be examined in the manual, semiautomatic, or automatic mode.

Figure 4 a shows a reconstructed ion image in MS mode for the parent ion at $m / z 1540$ that corresponds to pedal peptide with the sequence of PLDSVYGTHGMSGFA. Based on the quality of the MS mode peaks from the different positions in the sample-tailored geometry file, the AutoXecute feature of the Bruker FlexControl software automatically selects position/peak combinations for the acquisition of MS/MS spectra after the MS scan is finished. In this case, the thresholds for MS/MS acquisition were set so that the peak intensity must be higher than 400 counts and the signal-to-noise $>10$. The spatial distributions for two fragment ions corresponding to the parent ion at $m / z 1540$ are shown in Figure 4b, $m / z 419$, corresponding to the amino sequence PLDS with loss of ammonia and Figure 4c, $m / z$ 1245, corresponding to the sequence PLDSVYGTHGMS with loss of water. Not all of the neurons in this sample satisfied the signal quality requirements to produce $\mathrm{MS} / \mathrm{MS}$ spectra from the parent ion of $m / z 1540$, but the thresholds can be easily changed. Of course, many of the neurons used here would not contain appreciable amounts of pedal peptide and so would not be selected for MS/MS imaging; such cell-cell heterogeneity is one of the reasons that such individual cell assays are required.

Similarly, fragment ion distributions for a separate unidentified parent ion, $m / z$ 1200, are shown in Figure $4 \mathrm{e}$, f. This unknown compound was difficult to fragment and $\mathrm{MS} / \mathrm{MS}$ sequencing resulted in the generation of only a few fragments that were not identified. As the ability to fragment the compound at $m / z 1200$ varied between cells, perhaps this peak represents several isobaric analytes or effects of signal suppression by other cellular compounds. We have not identified this analyte, although the spatial distributions of the parent and fragment ions have been determined. Obtaining the spatial distributions of fragment ions allows detection and identification of the products of cell-specific enzymatic processing that often varies spatially, even among neighboring neurons [56]. Also, monitoring of specific cells producing MS/MS fragments can be important for imaging of the distribution of isobaric compounds.

Acquisition of analyte signals from neuronal somas cultured on bead substrates furthers the study of neuronal networks, including those cultured under different physicochemical conditions. The stretched imaging method protocols demonstrated here can be adapted to study neuronal network formation in a range of culturing conditions and from a variety of other model organisms. We will continue to refine these methods, significantly increasing their utility, 
as we extend our efforts toward collecting subcellular information from neuronal processes.

\section{Acknowledgments}

The authors thank C. Mark Bee, Sergei Dikler, Scott J. Robinson, Kevin R. Tucker, and Xiying Wang for discussions and experimental assistance. The project described was supported by Award nos. P30 DA018310 and 5RO1 DA017940 from the National Institute on Drug Abuse and Award no. 5RO1 DE018866 from the National Institute of Dental and Craniofacial Research (NIDCR) and the Office of Director (OD), National Institutes of Health (NIH). The content is solely the responsibility of the authors and does not necessarily represent the official views of the NIDA, NIDCR, or NIH.

\section{References}

1. Rahman, A.: The Role of Adenosine in Alzheimer's Disease. Curr. Neuropharmacol. 7, 207-216 (2009)

2. Kawane, K., Nagata, S.: Nucleases in Programmed Cell Death. Methods Enzymol. 442, 271-287 (2008)

3. Barar, J., Asadi, M., Mortazavi-Tabatabaei, S.A., Omidi, Y.: Ocular Drug Delivery; Impact of In Vitro Cell Culture Models. J. Ophthalmic Vis. Res. 4, 238-252 (2009)

4. Kirkpatrick, C.J., Fuchs, S., Hermanns, M.I., Peters, K., Unger, R.E. Cell Culture Models of Higher Complexity in Tissue Engineering and Regenerative Medicine. Biomaterials 28, 5193-5198 (2007)

5. Ogilvie, M.: Molecular Techniques Should Not Now Replace Cell Culture in Diagnostic Virology Laboratories. Rev. Med. Virol. 11, 351-354 (2001)

6. Athey, D., Shah, D.S.H., Phillips, S.R., Lakey, J.H.: A Manufacturable Surface-Biology Platform for Nano Applications; Cell Culture, Analyte Detection. Diagnostics Sensors. Ind. Biotechnol. 1, 185-189 (2005)

7. Sakudo, A., Nakamura, I., Ikuta, K., Onodera, T.: Recent Developments in Prion Disease Research: Diagnostic Tools and In Vitro Cell Culture Models. J. Vet. Med. Sci. 69, 329-337 (2007)

8. Gu, Y., Wang, J., Ding, F., Hu, N., Wang, Y., Gu, X.: Neurotrophic Actions of Bone Marrow Stromal Cells on Primary Culture of Dorsal Root Ganglion Tissues and Neurons. J. Mol. Neurosci. 40, 332-341 (2010)

9. Petiot, E., Bernard-Moulin, P., Magadoux, T., Gény, C., Pinton, H., Marc, A.: In Situ Quantification of Microcarrier Animal Cell Cultures Using Near-Infrared Spectroscopy. Process Biochem. 45, 1427-1431 (2010)

10. Sellick, C.A., Hansen, R., Jarvis, R.M., Maqsood, A.R., Stephens, G. M., Dickson, A.J., Goodacre, R.: Rapid Monitoring of Recombinant Antibody Production by Mammalian Cell Cultures Using Fourier Transform Infrared Spectroscopy and Chemometrics. Biotechnol. Bioeng. 106, 432-442 (2010)

11. Lage, H.: Proteomics in Cancer Cell Research: An Analysis of Therapy Resistance. Pathol. Res. Pract. 200, 105-117 (2004)

12. Woolley, J.F., Al-Rubeai, M.: The Application of SELDI-TOF Mass Spectrometry to Mammalian Cell Culture. Biotechnol. Adv. 27, 177184 (2009)

13. Zimmerman, T.A., Monroe, E.B., Tucker, K.R., Rubakhin, S.S., Sweedler, J.V.: Chapter 13: Imaging of Cells and Tissues with Mass Spectrometry: Adding Chemical Information to Imaging. Methods Cell Biol. 89, 361-390 (2008)

14. Francese, S., Dani, F.R., Traldi, P., Mastrobuoni, G., Pieraccini, G., Moneti, G.: MALDI Mass Spectrometry Imaging, from Its Origins up to Today: The State of the Art. Comb. Chem. High Throughput Screen. 12 156-174 (2009)

15. Rubakhin, S.S., Jurchen, J.C., Monroe, E.B., Sweedler, J.V.: Imaging Mass Spectrometry: Fundamentals and Applications to Drug Discovery. Drug Discov. Today 10, 823-837 (2005)

16. Andersson, M., Groseclose, M.R., Deutch, A.Y., Caprioli, R.M. Imaging Mass Spectrometry of Proteins and Peptides: 3D Volume Reconstruction. Nat. Methods 5, 101-108 (2008)
17. Stoeckli, M., Chaurand, P., Hallahan, D.E., Caprioli, R.M.: Imaging Mass Spectrometry: A New Technology for the Analysis of Protein Expression in Mammalian Tissues. Nat. Med. 7, 493-496 (2001)

18. Chaurand, P., Schwartz, S.A., Reyzer, M.L., Caprioli, R.M.: Imaging Mass Spectrometry: Principles and Potentials. Toxicol. Pathol. 33, 92-101 (2005)

19. Castaing, R., Slodzian, G.: Microanalyse Par Émission Ionique Secondaire. J. Microscop. 1, 395-410 (1962)

20. Heien, M.L., Piehowski, P.D., Winograd, N., Ewing, A.G.: Lipid Detection, Identification, and Imaging Single Cells with SIMS. Methods Mol. Biol. 656, 85-98 (2010)

21. Chandra, S.: Quantitative Imaging of Chemical Composition in Single Cells by Secondary Ion Mass Spectrometry: Cisplatin Affects Calcium Stores in Renal Epithelial Cells. Methods Mol. Biol. 656, 113-130 (2010)

22. Ishida, Y., Madonna, A.J., Rees, J.C., Meetani, M.A., Voorhees, K.J.: Rapid Analysis of Intact Phospholipids from Whole Bacterial Cells by Matrix-Assisted Laser Desorption/Ionization Mass Spectrometry Combined with On-Probe Sample Pretreatment. Rapid Commun. Mass Spectrom. 16, 1877-1882 (2002)

23. Rubakhin, S.S., Greenough, W.T., Sweedler, J.V.: Spatial Profiling with MALDI MS: Distribution of Neuropeptides within Single Neurons. Anal. Chem. 75, 5374-5380 (2003)

24. Claydon, M.A., Davey, S.N., Edwards-Jones, V., Gordon, D.B.: The Rapid Identification of Intact Microorganisms Using Mass Spectrometry. Nat. Biotechnol. 14, 1584-1586 (1996)

25. Bergquist, J.: Cells on the Target Matrix-Assisted Laser-Desorption/ Ionization Time-of-Flight Mass-Spectrometric Analysis of Mammalian Cells Grown on the Target. Chromatographia 49(Suppl. 1), S41-S48 (1999)

26. Northen, T. R.; Yanes, O.; Northen, M. T.; Marrinucci, D.; Uritboonthai, W.; Apon, J.; Golledge, S. L.; Nordström, A.; Siuzdak, G. Clathrate Nanostructures for Mass Spectrometry. Nature 2007, 1033-1036

27. Kruse, R.A., Rubakhin, S.S., Romanova, E.V., Bohn, P.W., Sweedler, J.V.: Direct Assay of Aplysia Tissues and Cells with Laser Desorption/ Ionization Mass Spectrometry on Porous Silicon. J. Mass Spectrom. 36, 1317-1322 (2001)

28. Shimma, S., Sugiura, Y., Hayasaka, T., Hoshikawa, Y., Nodae, T., Setou, M.: MALDI-Based Imaging Mass Spectrometry Revealed Abnormal Distribution of Phospholipids in Colon Cancer Liver Metastasis. J. Chromatogr. B 855, 98-103 (2007)

29. Yang, H.J., Sugiura, Y., Ikegami, K., Setou, M.: Imaging of Cultured Cells by Mass Spectrometry. In: Setou, M. (ed.) Imaging Mass Spectrometry: Protocols for Mass Microscopy, pp. 159-168. Springer, Japan (2010)

30. Rohner, T.C., Staaba, D., Stoeckli, M.: MALDI Mass Spectrometric Imaging of Biological Tissue Sections. Mech. Age Dev. 126, 177-185 (2005)

31. Klerk, L.A., Altelaar, A.F.M., Froesch, M., McDonnell, L.A., Heeren, R.M.A.: Fast and Automated Large-Area Imaging MALDI Mass Spectrometry in Microprobe and Microscope Mode. Int. J. Mass Spectrom. 285, 19-25 (2009)

32. Hazama, H., Aokib, J., Nagaoa, H., Suzukia, R., Tashima, T., Fujiic, K.-I., Masudad, K., Awazua, K., Toyodab, M., Naito, Y.: Construction of a Novel Stigmatic MALDI Imaging Mass Spectrometer. Appl. Surf. Sci. 255, 1257-1263 (2008)

33. Sherrod, S.D., Castellana, E.T., McLean, J.A., Russell, D.H.: Spatially Dynamic Laser Patterning Using Advanced Optics for Imaging Matrix Assisted Laser Desorption/Ionization (MALDI) Mass Spectrometry. Int. J. Mass Spectrom. 262, 256-262 (2007)

34. Hummon, A.B., Amare, A., Sweedler, J.V.: Discovering New Invertebrate Neuropeptides Using Mass Spectrometry. Mass Spectrom. Rev. 25, 77-98 (2006)

35. Rubakhin, S.S., Sweedler, J.V.: Quantitative Measurements of CellCell Signaling Peptides with Single-Cell MALDI MS. Anal. Chem. 80, $7128-7136$ (2008)

36. Zimmerman, T.A., Rubakhin, S.S., Romanova, E.V., Tucker, K.R., Sweedler, J.V.: MALDI Mass Spectrometric Imaging Using the Stretched Sample Method to Reveal Neuropeptide Distributions in Aplysia Nervous Tissue. Anal. Chem. 81, 9402-9409 (2009)

37. Monroe, E.B., Jurchen, J.C., Koszczuk, B.A., Losh, J.L., Rubakhin, S., Sweedler, J.V.: Massively Parallel Sample Preparation for the MALDI MS Analyses of Tissue. Anal. Chem 78, 6832 (2006)

38. Zimmerman, T.A., Rubakhin, S.S., Sweedler, J.V.: Mass Spectrometry Imaging Using the Stretched Sample Approach. Methods Mol. Biol. 656, 465-479 (2010) 
39. Li, L., Moroz, T.P., Garden, R.W., Floyd, P.D., Weiss, K., Sweedler, J. V.: Mass Spectrometric Survey of Interganglionically Transported Peptides in Aplysia. Peptides 19, 1425-1433 (1998)

40. Zimmerman, T.A., Monroe, E.B., Sweedler, J.V.: Adapting the Stretched Sample Method from Tissue Profiling to Imaging. Proteomics 8, 3809-3815 (2008)

41. Rubakhin, S.S., Hatcher, N.G., Monroe, E.B., Heien, M.L., Sweedler, J. V.: Mass Spectrometric Imaging of the Nervous System. Curr. Pharm. Des. 13, 3325-3334 (2007)

42. Schmidt, C.E., Leach, J.B.: Neural Tissue Engineering: Strategies for Repair and Regeneration. Annu. Rev. Biomed. Eng. 5, 293-347 (2003)

43. Saito, T., Hayashib, H., Kameyama, T., Hishida, M., Nagai, K., Teraoka, K., Kato, K.: Suppressed Proliferation of Mouse OsteoblastLike Cells by a Rough-Surfaced Substrate Leads to Low Differentiation and Mineralization. Mater. Sci. Eng. C 30, 1-7 (2010)

44. Kratje, R.B., Reimann, A., Hammer, J., Wagner, R.: Cultivation of Recombinant Baby Hamster Kidney Cells in a Fluidized Bed Bioreactor System with Porous Borosilicate Glass. Biotechnol. Prog. 10, 410-420 (1994)

45. Collazos-Castro, J.E., Cruz, A.M., Carballo-Vila, M., Lira-Cantú, M., Abad, L., Pino, Á.P.D., Fraxedas, J., Juan, A.S., Fonseca, C., Pêgo, A. P., Casañ-Pastor, N.: Neural Cell Growth on TiO2 Anatase Nanostructured Surfaces. Thin Solid Films 518, 160-170 (2009)

46. Wei, H., Li, H., Gao, D., Lin, J.-M.: Multi-Channel Microfluidic Devices Combined with Electrospray Ionization Quadrupole Time-ofFlight Mass Spectrometry Applied to the Monitoring of Glutamate Release from Neuronal Cells. Analyst 135, 2043-2050 (2010)

47. Atashi, A., Nadri, S., Hafizi, M., Soleimani, M.: Role of Poly-L-LysineCoated Plates and Fetal Calf Serum Concentration in Sheep Chondroprogenitor Cell Culturing. J. Artif. Organs 12, 118-122 (2009)

48. Hanson, J.N., Motola, M.J., Heien, M.L., Gillette, M., Sweedler, J.V., Nuzzo, R.G.: Textural Guidance Cues for Controlling Process Outgrowth of Mammalian Neurons. Lab Chip 9, 122-131 (2009)
49. Zhang, J., Venkataramani, S., Xua, H., Song, Y.-K., Song, H.-K., Palmore, G.T.R., Fallonc, J., Nurmikko, A.V.: Combined Topographical and Chemical Micropatterns for Templating Neuronal Networks. Biomaterials 27, 5734-5739 (2006)

50. Yu, L.M.Y., Leipzig, N.D., Shoicheta, M.S.: Promoting Neuron Adhesion and Growth. Mater. Today 11, 36-43 (2008)

51. Hatada, Y., Wu, F., Silverman, R., Schacher, S., Goldberg, D.J.: En Passant Synaptic Varicosities Form Directly from Growth Cones by Transient Cessation of Growth Cone Advance but Not of Actin-Based Motility. J. Neurobiol. 41, 242-251 (1999)

52. Chen, R., Jiang, X., Conaway, M.C.P., Mohtashemi, I., Hui, L., Viner, R., Li, L.: Mass Spectral Analysis of Neuropeptide Expression and Distribution in the Nervous System of the Lobster Homarus americanus. J. Proteome Res. 9, 818-832 (2010)

53. Drexler, D.M., Garrett, T.J., Cantone, J.L., Diters, R.W., Mitroka, J.G., Conaway, M.C.P., Adams, S.P., Yost, R.A., Sanders, M.: Utility of Imaging Mass Spectrometry (IMS) by Matrix-Assisted Laser Desorption Ionization (MALDI) on an Ion Trap Mass Spectrometer in the Analysis of Drugs and Metabolites in Biological Tissues. J. Pharmacol. Toxicol. Methods 55, 279-288 (2007)

54. Verhaert, P.D.E.M., Pinkse, M.W.H., Strupat, K., Conaway, M.C.P.: Imaging of Similar Mass Neuropeptides in Neuronal Tissue by Enhanced Resolution MALDI MS with an Ion Trap-Orbitrap Hybrid Instrument. Methods Mol. Biol. 656, 433-449 (2010)

55. Scherl, A., Zimmermann-Ivol, C.G., Dio, J.D., Vaezzadeh, A.R., Binz, P.-A., Amez-Droz, M., Cochard, R., Sanchez, J.-C., Glückmann, M., Hochstrasse, D.F.: Gold Coating of Nonconductive Membranes Before Matrix-Assisted Laser Desorption/Ionization Tandem Mass Spectrometric Analysis Prevents Charging Effect. Rapid Commun. Mass Spectrom. 19, 605-610 (2005)

56. Romanova, E.V., McKay, N., Weiss, K.R., Sweedler, J.V., Koester, J.: Autonomic Control Network Active in Aplysia During Locomotion Includes Neurons that Express Splice Variants of R15-Neuropeptides. $J$. Neurophysiol. 97, 481-491 (2007) 